\title{
Avaliação do enfoque familiar e orientação para a comunidade na Estratégia Saúde da Família
}

\author{
Evaluation of the family focus and community orientation \\ in the Family Health Strategy
}

Monyk Neves de Alencar ${ }^{1}$

Liberata Campos Coimbra ${ }^{1}$

Ana Patrícia Pereira Morais ${ }^{2}$

Antônio Augusto Moura da Silva ${ }^{1}$

Siane Rocha de Almeida Pinheiro ${ }^{1}$

Rejane Christine de Sousa Queiroz ${ }^{1}$

${ }^{1}$ Departamento de Saúde Pública, Centro de Ciências da Saúde, Universidade Federal do Maranhão. Rua Barão de Itapary 15, Centro. 65.020-070 São Luís MA Brasil.

monykneves@yahoo.com.br

${ }^{2}$ Departamento de Enfermagem, Centro de Ciências da Saúde, Universidade Estadual do Ceará.

\begin{abstract}
The Family Health Strategy should be focused on the family unit and constructed operationally within the community sphere. The research assessed the family focus and community orientation as attributes of Primary Health Care, comparing if the responses differed among users, professionals and managers. It is an evaluative study of a population-based quantitative approach conducted between January 2010 and March 2011 in São Luis in the state of Maranhão. The study involved a population of 32 managers and 80 professionals with more than six months experience in the Family Health Strategy, and 883 users were selected by means of cluster sampling. Questionnaires validated in Brazil were used based on the components of the Primary Care Assessment Tool (PCATool). The composite index of the family focus was 2.7 for users, 4.9 for professionals and 5.3 for managers. In the posttest phase, differences were detected between users and professionals, and users and managers. The composite index of community orientation was 2.9 for users, 3.9 for professionals and 4.8 for managers $(p<0.001)$. Managers attributed higher percentages in all indicators, followed by professionals and lastly users. Both attributes were rated as being unsatisfactory in the perception of the users.
\end{abstract}

Key words Primary care, Health evaluation, Family Health Strategy
Resumo A Estratégia Saúde da Família deve ser focada na unidade familiar e construída operacionalmente na esfera comunitária. A presente pesquisa avaliou o enfoque familiar e a orientação para a comunidade como atributos da Atenção Primária em Saúde, comparando se as respostas diferem entre usuários, profissionais e gestores. Trata-se de pesquisa avaliativa de abordagem quantitativa de base populacional, realizada de janeiro de 2010 a março de 2011 no município de São Luís (MA). Trabalhou-se com população de 32 gestores e 80 profissionais com mais de seis meses de experiência na Estratégia e com amostra de 883 usuários selecionados por conglomerados. Foram utilizados questionários validados no Brasil a partir dos componentes do Primary Care Assessment Tool (PCATool). O indice composto do enfoque familiar foi de 2,7 para os usuários, 4,9 para os profissionais e 5,3 para os gestores. No pós-teste as diferenças foram entre os usuários e os profissionais, e usuários e gestores. $O$ indice composto da orientação para a comunidade foi de 2,9 para os usuários, 3,9 para os profissionais e 4,8 para os gestores $(p<0,001)$. Os gestores atribuiram percentuais mais altos em todos os indicadores, depois os profissionais e por último os usuários. Os dois atributos tiveram avaliação insatisfatória na percepção dos usuários.

Palavras-chave Atenção primária, Avaliação em saúde, Estratégia Saúde da Família 


\section{Introdução}

No Brasil, a Atenção Básica à Saúde (ABS) representa o primeiro nível de atenção à saúde $\mathrm{e}$ sustenta-se no princípio da integralidade, compreendido como a articulação de ações de promoção da saúde e prevenção, tratamento e reabilitação de doenças e agravos. O conceito usual para este campo de práticas no restante do mundo é Atenção Primária à Saúde (APS), como decorrência do pacto mundial realizado em 1978, constituindo na Declaração de Alma-Ata ${ }^{1}$.

Acredita-se que APS é uma estratégia eficaz de melhoria dos resultados de saúde em virtude do aumento do acesso aos serviços básicos, pelo impacto resultante sob a justiça social e no processo de empoderamento das comunidades alvo. Entretanto, para serem efetivos, sistemas de APS devem ser baseados no conhecimento científico acumulado e utilizar métodos socialmente e culturalmente aceitos, que possam estar disponíveis para os indivíduos e as famílias na comunidade onde residam ${ }^{2}$.

A Estratégia de Saúde da Família (ESF), desde sua origem, foi concebida para a reorganização e o fortalecimento da APS como o primeiro nível de atenção à saúde no SUS, mediante a ampliação do acesso, a qualificação e a reorientação das suas práticas ${ }^{1}$. É um modelo de atenção focado na unidade familiar e construído operacionalmente na esfera comunitária.

$\mathrm{Na}$ discussão sobre a avaliação dos programas de atenção primária Starfield ${ }^{3}$ discorre sobre a qualidade destes serviços, destacando como atributos derivados a centralização na família, a orientação para a comunidade e a competência cultural.

O enfoque na família acontece quando o alcance da integralidade fornece uma base para a consideração dos indivíduos dentro de seus ambientes, quando a avaliação das necessidades para a atenção integral considera o contexto familiar e sua exposição a ameaças à saúde e quando o desafio da coordenação da atenção se defronta com recursos familiares limitados ${ }^{3}$.

Uma abordagem orientada para a comunidade aplica os métodos da medicina clínica, epidemiologia, ciências sociais e pesquisa e avaliação de serviços de saúde para as tarefas de: definir e caracterizar a comunidade; identificar seus problemas de saúde; modificar programas para abordar estes problemas e monitorar a efetividade dessas modificações ${ }^{3}$.

Para o fortalecimento e a consolidação de uma APS de qualidade é de suma importância desen- volver e aprimorar mecanismos de avaliação da organização, do desempenho e dos resultados dessa abordagem e engendrar esforços no sentido de trazer a avaliação à rotina dos serviços e institucionalizá-la ${ }^{4}$.

A avaliação deve ser concebida como uma ferramenta habitual indispensável à formulação da própria ação, de sua implementação e melhoria. Ela tem várias dimensões: primeiro, uma cognitiva, a avaliação visa produzir informações científicas válidas e socialmente legítimas; segundo, tem uma dimensão normativa quando visa a emitir um julgamento e, terceiro, uma dimensão instrumental ou utilitária quando visa a melhorar uma situação ${ }^{5}$.

Alguns estudos têm feito avaliação da atenção básica utilizando como objeto todos os atributos que caracterizam um serviço de saúde de atenção primária. Os atributos enfoque familiar e a orientação para a comunidade têm recebido os piores escores de avaliação ${ }^{6-8}$.

Em estudos que compararam as Unidades de Saúde da Família (USF) com outras unidades básicas, estes dois atributos aparecem com melhor desempenho nas unidades da ESF, entretanto, com escores baixos e aquém do esperado, considerando os princípios e as diretrizes da Saúde da Família ${ }^{7,8}$. Não há trabalhos que analisem os indicadores destes dois atributos como características destes serviços, pois os estudos enfocam apenas o escore total.

Avaliar a qualidade dos serviços, principalmente focando na atenção à saúde centrada na família e na orientação comunitária, constitui-se uma prioridade no atual processo de expansão e consolidação da Estratégia. Portanto, este estudo tem como objetivo avaliar o enfoque familiar e a orientação para a comunidade como atributos da APS na perspectiva de três grupos: usuários, profissionais e gestores da ESF.

\section{Métodos}

\section{Tipo, local e período do estudo}

Trata-se de uma pesquisa avaliativa de abordagem quantitativa realizada no período de janeiro de 2010 a março de 2011 no município de São Luís (MA). À época do estudo, no ano de 2009, o município contava com 78 equipes da ESF registradas no Cadastro Nacional de Estabelecimentos de Saúde (CNES), distribuídas em 39 unidades de saúde, com cobertura de 30,3\% da população?. 
Os sujeitos deste estudo foram gestores, profissionais e usuários da ESF em São Luís (MA). Todos os gestores e profissionais compuseram a população estudada, sendo, portanto, 52 gestores (um coordenador da ABS, 12 supervisores de áreas e 39 diretores das USF) e 156 profissionais (78 médicos e 78 enfermeiros). O critério de inclusão dos gestores e profissionais foi que tivessem pelo menos seis meses de experiência na ESF.

Para a seleção dos usuários, calculou-se o tamanho amostral, tendo como parâmetros alfa 0,05 e poder do teste 0,8 . Foram acrescidos $10 \%$ para cobrir possíveis perdas, totalizando uma amostra de 917. No cálculo foi necessário controlar a correlação intraclasse, multiplicando-se o tamanho da amostra pelo efeito do desenho. Utilizou-se como referência para o cálculo do valor da correlação o trabalho de Almeida e Macinko ${ }^{10}, \mathrm{p}=0,25$.

Os usuários foram selecionados por meio de amostragem por conglomerados em duas etapas. Inicialmente foram selecionadas aleatoriamente 20 das 39 unidades de saúde da ESF. Nestas 20 existiam 44 equipes da ESF em funcionamento. Para se obter o número de usuários entrevistados por equipe dividiu-se o tamanho amostral pelo número de equipes e obteve-se 20,8, o que foi aproximado para 21 aumentando o número total de entrevistas esperadas para 924.

Os critérios de inclusão dos usuários foram: ter pelo menos 18 anos de idade, estar cadastrado na ESF há pelo menos seis meses e ter sido atendido anteriormente pela equipe. Foram ainda incluídas todas as gestantes e mulheres que já tiveram filhos, independente da sua idade. Para os usuários menores de idade ou incapacitados o respondente do questionário foi o acompanhante, que deveria já ter acompanhado o usuário na unidade em outra oportunidade e ter pelo menos 18 anos, com exceção das mães acompanhando crianças.

\section{Instrumentos de Pesquisa}

Foram utilizados questionários validados no Brasil a partir dos componentes do Primary Care

Assessment Tool (PCATool), instrumento formulado para avaliar os aspectos críticos da atenção primária, mensurados por meio de escores, baseado no modelo de avaliação da qualidade de serviços de saúde proposto por Donabedian que mensura aspectos de estrutura, processo e resultado dos serviços de saúde $e^{10,11}$.

Foram aplicados quatro tipos de questionários de acordo com o respondente: gestores, profissionais e usuários/acompanhantes. O questionário foi dividido em oito seções, duas delas se referiam aos atributos "enfoque familiar" e "orientação para a comunidade”.

Os indicadores investigados sobre o enfoque familiar foram: organização dos prontuários médicos por família; solicitação de informações sobre doenças de outros membros da família pela equipe; disponibilidade dos profissionais de saúde para falar com a família do usuário; questionamento pelos profissionais de saúde durante a anamnese sobre fatores de risco social e condições de vida do usuário e a possibilidade do usuário expressar sua opinião sobre seu tratamento.

Os indicadores relacionados à orientação para a comunidade foram: realização de enquetes com os usuários para identificar problemas de saúde da população e se os serviços atendem suas necessidades; participação da comunidade na direção das USF; presença de serviços de saúde oferecidos nas escolas; realização de visitas domiciliares e existência de programas intersetoriais com outras instituições ou organizações.

Os entrevistados responderam a cada pergunta segundo uma escala de possibilidades preestabelecida, escala Likert de respostas: 1 (nunca), 2 (quase nunca), 3 (algumas vezes), 4 (muitas vezes), 5 (quase sempre), 6 (sempre) e não sabe. Quanto maior o percentual e o escore, melhor a avaliação dos atributos estudados.

\section{Coleta de dados}

Os dados foram coletados por equipe treinada em todas as etapas da pesquisa. Foi realizado estudo piloto em uma unidade de saúde do município, o que possibilitou ajustes.

O coordenador da ABS e os supervisores de áreas da ESF foram entrevistados na Secretaria Municipal de Saúde, enquanto os profissionais e gestores locais, na própria unidade. A entrevista com os usuários foi realizada nas USF em dias e turnos alternados, antes ou após o atendimento pela equipe. 


\section{Análise dos dados}

Foram criadas tabelas com os percentuais de respostas dos três grupos para todos os indicadores dos dois atributos. Todos os escores dos indicadores sobre enfoque familiar foram somados e a média dessa soma representou o Índice Composto do Enfoque Familiar. Esse mesmo processo foi seguido para construir o Índice Composto da Orientação para a Comunidade.

Para detectar a existência de diferenças estatisticamente significantes nas respostas dos usuários, profissionais e gestores foi utilizado o teste de Kruskal-Wallis e entre profissionais e gestores utilizou-se o teste de Mann-Whitney. Para identificar entre quais grupos estudados estavam as diferenças encontradas foi aplicado o pós-teste não paramétrico para comparações múltiplas, por meio do comando kwallis2 no STATA. O ponto de corte para significância estatística foi definido em $5 \%(p<0,05)$. Foram utilizados os softwares EPI-INFO 3.2 e STATA 10.0 para processamento e análise dos dados.

\section{Aspectos Éticos}

A Pesquisa foi aprovada pelo Comitê de Ética em Pesquisa da Universidade Federal do Maranhão. As entrevistas foram iniciadas somente após a leitura, concordância e assinatura do Termo de Consentimento Livre e Esclarecido, respeitando-se os princípios éticos e legais da Resolução no $196 / 96^{12}$ e suas complementares.

\section{Resultados}

Participaram do estudo 80 profissionais e 32 gestores. Não atenderam aos critérios de inclusão $12,7 \%$ dos profissionais e $12,8 \%$ dos gestores. Foram consideradas perdas: $15,8 \%$ dos profissionais e $7,7 \%$ dos gestores que se recusaram; $14,5 \%$ dos profissionais e $15,4 \%$ dos gestores com os quais não foi conseguido contato; e 6,4\% dos profissionais que estavam de licença ou férias.

Dos profissionais entrevistados, 72,5\% eram enfermeiros e $27,5 \%$ médicos, 93,8\% tinham especialização como maior titulação e $62,4 \%$ tinham experiência de 13 a 48 meses na ESF. Quanto aos gestores, 62,5\% eram diretores das Unidades de Saúde, 31,3\% coordenadores de área da ESF e $6,2 \%$ coordenadores centrais da Secretaria $\mathrm{Mu}$ nicipal de Saúde (SEMUS). Em relação ao tempo de experiência na função, 56,3\% trabalhavam de 6 a 12 meses. Sobre a titulação acadêmica: 71,9\% tinham especialização, 15,6\% eram graduados e $9,4 \%$ tinham grau de mestre.

Foram entrevistados 883 usuários, destes 234 eram acompanhantes de usuários menores de idade ou incapacitados. Houve perda de 4,5\%, visto que uma unidade não estava funcionando no período da coleta.

Verificou-se que 72,6\% dos usuários eram do sexo feminino; $32 \%$ tinham ensino fundamental incompleto, seguido de 25,3\% com ensino médio completo; $21,6 \%$ encontravam-se na faixa etária de 20 a 29 anos de idade e 18,3\% tinham de zero a nove anos. A média de idade dos usuários foi de 32,1 anos.

Os percentuais de obtenção das respostas nunca, quase nunca, algumas vezes, muitas vezes, quase sempre, sempre e não sabe encontramse na Tabela 1 para o atributo enfoque familiar e na Tabela 2 para o enfoque orientação para a comunidade. Os percentuais de nunca e quase nunca foram maiores para os usuários, menores para os profissionais e menores ainda para os gestores em quase todos os indicadores. Vale ressaltar que 57,8\% dos usuários não souberam informar a existência de estímulo da secretaria municipal para a participação de representantes da comunidade em reuniões de direção da USF ou no conselho local de saúde, bem como 54\% não souberam responder se há oferta de serviços nas escolas pelas equipes de ESF.

Na Tabela 3 estão dispostos os percentuais de obtenção considerados ideais para cada tipo de atributo (quase sempre e sempre) segundo os três grupos: gestores profissionais e usuários. Há diferenças estatisticamente significantes $(\mathrm{p}<$ $0,001)$ entre as percepções dos três grupos em todos os indicadores do enfoque familiar e da orientação para a comunidade, com maiores percentuais para os gestores e menores para os usuários. Segundo os usuários, em 16,7\% das consultas os profissionais perguntam sobre os seus fatores de risco social ou condições de vida, enquanto para os gestores isto ocorre em percentual maior, $81,2 \%$ das vezes. Apenas 13,9\% dos usuários informaram que quase sempre ou sempre são questionados pelos profissionais sobre suas ideias e opiniões acerca do seu tratamento.

A Tabela 4 apresenta os escores dos indicadores e o índice composto para os dois atributos estudados. $\mathrm{O}$ índice composto do enfoque familiar foi de 2,7 para os usuários, 4,9 para os profissionais e 5,3 para os gestores. Observaram-se diferenças estatisticamente significantes entre os três grupos $(\mathrm{p}<0,001)$. No pós-teste a diferença mostrou-se na percepção entre usuários e pro- 
Tabela 1. Percepção dos usuários, profissionais e gestores da Estratégia Saúde da Família sobre o atributo enfoque familiar no município de São Luís - MA, 2011.

\begin{tabular}{|c|c|c|c|c|c|c|c|}
\hline Indicadores & $\begin{array}{l}\text { Nunca } \\
\%\end{array}$ & $\begin{array}{l}\text { Quase } \\
\text { nunca } \\
\%\end{array}$ & $\begin{array}{l}\text { Algumas } \\
\text { vezes } \\
\%\end{array}$ & $\begin{array}{c}\text { Muitas } \\
\text { vezes } \\
\%\end{array}$ & $\begin{array}{l}\text { Quase } \\
\text { sempre } \\
\%\end{array}$ & $\begin{array}{l}\text { Sempre } \\
\quad \%\end{array}$ & $\begin{array}{l}\text { Não } \\
\text { sabe } \\
\%\end{array}$ \\
\hline \multicolumn{8}{|l|}{ Usuários（ $\mathrm{n}=883$ ) } \\
\hline $\begin{array}{l}\text { Questionamento dos profissionais } \\
\text { sobre fatores de risco social ou } \\
\text { condições de vida dos usuários }\end{array}$ & 52,0 & 9,1 & 14,8 & 7,1 & 6,3 & 10,4 & 0,1 \\
\hline $\begin{array}{l}\text { Questionamento sobre doenças de } \\
\text { familiares durante a consulta }\end{array}$ & 35,4 & 8,0 & 16,1 & 8,1 & 7,2 & 24,0 & 1,1 \\
\hline $\begin{array}{l}\text { Conhecimento da família pelos } \\
\text { profissionais }\end{array}$ & 45,6 & 13,1 & 14,0 & 7,7 & 6,5 & 8,3 & 4,6 \\
\hline $\begin{array}{l}\text { Possibilidade do profissional discutir } \\
\text { com a família problemas de saúde } \\
\text { dos usuários }\end{array}$ & 21,7 & 6,4 & 15,8 & 11,1 & 10,8 & 20,5 & 13,4 \\
\hline $\begin{array}{l}\text { Questionamento dos profissionais } \\
\text { sobre ideias e opiniões dos usuários } \\
\text { acerca do seu tratamento }\end{array}$ & 59,0 & 11,3 & 9,6 & 3,8 & 4,1 & 9,8 & 2,2 \\
\hline \multicolumn{8}{|l|}{ Profissionais $(\mathrm{n}=80)$} \\
\hline $\begin{array}{l}\text { Questionamento sobre doenças de } \\
\text { familiares durante a consulta }\end{array}$ & - & 2,5 & 12,5 & 17,5 & 30,0 & 37,5 & - \\
\hline $\begin{array}{l}\text { Possibilidade do profissional discutir } \\
\text { com a família problemas de saúde } \\
\text { dos usuários }\end{array}$ & 1,2 & 5,0 & 20,0 & 20,0 & 27,0 & 26,2 & - \\
\hline $\begin{array}{l}\text { Questionamento dos profissionais } \\
\text { sobre fatores de risco social ou } \\
\text { condições de vida dos usuários }\end{array}$ & - & 6,2 & 16,2 & 18,7 & 22,5 & 36,2 & - \\
\hline $\begin{array}{l}\text { Organização dos prontuários médicos } \\
\text { por família na UBS }\end{array}$ & 2,5 & - & - & 1,2 & 15,0 & 77,5 & 3,7 \\
\hline \multicolumn{8}{|l|}{ Gestores $(\mathrm{n}=32)$} \\
\hline $\begin{array}{l}\text { Questionamento sobre doenças de } \\
\text { familiares durante a consulta }\end{array}$ & - & - & 6,2 & 12,5 & 28,1 & 53,1 & - \\
\hline $\begin{array}{l}\text { Possibilidade de o profissional } \\
\text { discutir com a família problemas de } \\
\text { saúde dos usuários }\end{array}$ & - & - & 6,2 & 21,8 & 28,1 & 43,7 & - \\
\hline $\begin{array}{l}\text { Questionamento dos profissionais } \\
\text { sobre fatores de risco social ou } \\
\text { condições de vida dos usuários }\end{array}$ & - & 3,1 & 3,1 & 12,5 & 31,2 & 50,0 & - \\
\hline $\begin{array}{l}\text { Organização dos prontuários médicos } \\
\text { por família na UBS }\end{array}$ & - & - & - & 3,1 & - & 96,8 & - \\
\hline
\end{tabular}

fissionais e usuários e gestores $(\mathrm{p}<0,001)$. Não houve diferenças estatisticamente significantes entre os profissionais e gestores.

O índice composto da orientação para a comunidade foi de 2,9 para os usuários, 3,9 para os profissionais e 4,8 para os gestores. Observaramse diferenças estatisticamente significantes entre os três grupos $(\mathrm{p}<0,001)$. No pós-teste alguns indicadores apresentaram diferenças nas percepções dos três grupos $(\mathrm{p}<0,05)$. Não houve dife- renças estatísticas entre os profissionais e gestores com relação à frequência de visitas domiciliares e quanto à realização de ações intersetoriais.

\section{Discussão}

As análises evidenciam que as percepções dos gestores em todos os indicadores estudados estiveram sempre em percentuais e escores mais ele- 
Tabela 2. Percepção dos usuários, profissionais e gestores da Estratégia Saúde da Família sobre o atributo orientação para a comunidade no município de São Luís - MA, 2011.

\begin{tabular}{|c|c|c|c|c|c|c|c|}
\hline Indicadores & $\begin{array}{c}\text { Nunca } \\
\%\end{array}$ & $\begin{array}{l}\text { Quase } \\
\text { nunca } \\
\%\end{array}$ & $\begin{array}{l}\text { Algumas } \\
\text { vezes } \\
\%\end{array}$ & $\begin{array}{l}\text { Muitas } \\
\text { vezes } \\
\%\end{array}$ & $\begin{array}{l}\text { Quase } \\
\text { sempre } \\
\%\end{array}$ & $\begin{array}{l}\text { Sempre } \\
\%\end{array}$ & $\begin{array}{l}\text { Não } \\
\text { sabe } \\
\%\end{array}$ \\
\hline \multicolumn{8}{|l|}{ Usuários $(\mathrm{n}=883)$} \\
\hline $\begin{array}{l}\text { Família é consultada para saber se os serviços da USB } \\
\text { atendem aos problemas de saúde dos usuários }\end{array}$ & 56,3 & 11,1 & 13,3 & 3,8 & 6,0 & 8,6 & 1,0 \\
\hline $\begin{array}{l}\text { Conhecimentos dos profissionais sobre os principais } \\
\text { problemas de saúde da comunidade }\end{array}$ & 19,8 & 10,4 & 17,5 & 11,1 & 10,7 & 15,0 & 15,3 \\
\hline $\begin{array}{l}\text { Estimulo da SEMUS para a participação de } \\
\text { representantes da comunidade em reuniões de } \\
\text { direção da USB ou conselho local }\end{array}$ & 13,7 & 5,3 & 8,5 & 2,8 & 3,6 & 8,1 & 57,8 \\
\hline Oferta de serviços nas escolas pela equipe da ESF & 17,2 & 5,3 & 9,1 & 2,7 & 3,4 & 8,7 & 54,0 \\
\hline Frequência de visitas domiciliares & 15,0 & 19,5 & 23,4 & 8,1 & 11,6 & 18,0 & 5,1 \\
\hline $\begin{array}{l}\text { Trabalho com outros grupos para realizar atividades } \\
\text { que melhore as condições de vida da comunidade }\end{array}$ & 15,3 & 8,5 & 10,0 & 3,2 & 7,0 & 8,5 & 47,5 \\
\hline \multicolumn{8}{|l|}{ Profissionais $(\mathrm{n}=80)$} \\
\hline $\begin{array}{l}\text { Realização de enquetes para saber se os serviços } \\
\text { oferecidos estão respondendo às necessidades da } \\
\text { população }\end{array}$ & 25,0 & 31,2 & 20,0 & 8,7 & 3,7 & 7,5 & 3,7 \\
\hline $\begin{array}{l}\text { Realização de enquetes para identificar problemas de } \\
\text { saúde da população }\end{array}$ & 23,7 & 23,7 & 25,0 & 10,0 & 11,2 & 3,7 & 2,5 \\
\hline $\begin{array}{l}\text { Representação da comunidade na direção da UBS ou } \\
\text { nos conselhos }\end{array}$ & 22,5 & 6,2 & 11,2 & 5,0 & 13,7 & 32,5 & 8,7 \\
\hline Oferta de serviços nas escolas pela equipe da ESF & 2,5 & 6,2 & 15,0 & 26,2 & 18,7 & 28,7 & 2,5 \\
\hline Frequência de visitas domiciliares & - & - & 2,5 & 5,0 & 20,0 & 72,5 & - \\
\hline $\begin{array}{l}\text { Trabalho da USF com outras instituições da } \\
\text { comunidade para realizar programas intersetoriais }\end{array}$ & 6,2 & 15,0 & 18,7 & 16,2 & 20,0 & 20,0 & 3,7 \\
\hline $\begin{array}{l}\text { Autonomia da USF para organizar a oferta de } \\
\text { serviços de acordo com os problemas da comunidade }\end{array}$ & 5,0 & 8,7 & 11,2 & 16,2 & 15,0 & 40,0 & 3,7 \\
\hline \multicolumn{8}{|l|}{ Gestores $(\mathrm{n}=32)$} \\
\hline $\begin{array}{l}\text { Realização de enquetes para saber se os serviços } \\
\text { oferecidos estão respondendo às necessidades da } \\
\text { população }\end{array}$ & 6,2 & 9,4 & 15,6 & 25,0 & 15,6 & 28,1 & - \\
\hline $\begin{array}{l}\text { Realização de enquetes para identificar problemas de } \\
\text { saúde da população }\end{array}$ & 3,1 & 9,4 & 18,7 & 18,7 & 25,0 & 25,0 & - \\
\hline $\begin{array}{l}\text { Representação da comunidade na direção da UBS ou } \\
\text { nos conselhos }\end{array}$ & 3,1 & 9,4 & 6,2 & 6,2 & 15,6 & 59,3 & - \\
\hline Oferta de serviços nas escolas pela equipe da ESF & - & 3,1 & 6,2 & 15,6 & 15,6 & 59,3 & - \\
\hline Frequência de visitas domiciliares & - & - & - & 6,2 & 3,1 & 87,5 & 3,10 \\
\hline $\begin{array}{l}\text { Trabalho da USF com outras instituições da } \\
\text { comunidade para realizar programas intersetoriais }\end{array}$ & 3,1 & 3,1 & 21,8 & 9,3 & 32,1 & 31,2 & - \\
\hline $\begin{array}{l}\text { Autonomia da USF para organizar a oferta de } \\
\text { serviços de acordo com os problemas da comunidade }\end{array}$ & - & 6,2 & 12,5 & 9,4 & 21,8 & 50,0 & \\
\hline
\end{tabular}

vados em comparação aos outros dois grupos estudados, principalmente em relação aos usuários. Observa-se grande discrepância entre a opinião de quem está na gestão e dos que utilizam de fato o serviço. Essas significativas diferenças encontradas entre os sujeitos corroboram com outros estudos que utilizaram o mesmo instrumento de pesquisa ${ }^{6,7,8,13}$.
$\mathrm{Na}$ avaliação dos usuários há pouco ou nenhum interesse dos profissionais de saúde da ESF quanto às suas condições de vida e de seus familiares, se caracterizando, por exemplo, pela inexistência de questionamentos sobre fatores de risco social e ausência de discussões com outros membros da família sobre os problemas de saúde encontrados. Já na percepção dos profissio- 
Tabela 3. Análise das percepções dos usuários, profissionais e gestores da Estratégia Saúde da Família quanto ao melhor desempenho dos indicadores do enfoque familiar e da orientação para a comunidade no município de São Luís - MA, 2011.

\begin{tabular}{|c|c|c|c|c|}
\hline Indicadores & $\begin{array}{c}\text { Usuários }(\mathbf{n}=\mathbf{8 8 3}) \\
\text { Quase sempre/Sempre } \\
(\text { IC } 95 \%)\end{array}$ & $\begin{array}{l}\text { Profissionais }(\mathbf{n}=\mathbf{8 0}) \\
\text { Quase sempre/Sempre } \\
\text { (IC 95\%) }\end{array}$ & $\begin{array}{c}\text { Gestores }(n=32) \\
\text { Quase sempre/Sempre } \\
(\text { IC } 95 \%)\end{array}$ & P-valor \\
\hline \multicolumn{5}{|l|}{ Enfoque familiar } \\
\hline $\begin{array}{l}\text { Questionamento dos } \\
\text { profissionais sobre fatores de } \\
\text { risco social ou condições de } \\
\text { vida dos usuários }\end{array}$ & $16,7(13,6-18,5)$ & $58,7(46-68,4)$ & $81,2(63,5-92,7)$ & $<0,001^{*}$ \\
\hline $\begin{array}{l}\text { Questionamento sobre } \\
\text { doenças de familiares durante } \\
\text { a consulta }\end{array}$ & $31,2(28-34,1)$ & $67,5(53,5-75,3)$ & $74,2(56,5-88,5)$ & $<0,001^{*}$ \\
\hline $\begin{array}{l}\text { Conhecimento da família } \\
\text { pelos profissionais }\end{array}$ & $14,8(11,8-16,5)$ & - & - & \\
\hline $\begin{array}{l}\text { Possibilidade do profissional } \\
\text { discutir com a família } \\
\text { problemas de saúde dos } \\
\text { usuários }\end{array}$ & $31,3(28-34,1)$ & $53,2(41-63,7)$ & $71,8(53,2-86,2)$ & $<0,001^{*}$ \\
\hline $\begin{array}{l}\text { Questionamento dos } \\
\text { profissionais sobre ideias e } \\
\text { opiniões dos usuários acerca } \\
\text { do seu tratamento }\end{array}$ & $13,9(10,8-15,4)$ & - & - & \\
\hline $\begin{array}{l}\text { Organização dos prontuários } \\
\text { médicos por família na UBS }\end{array}$ & - & $92,5(84,3-97,1)$ & $96,8(83,7-100)$ & 0,671 \\
\hline \multicolumn{5}{|l|}{ Orientação para a comunidade } \\
\hline $\begin{array}{l}\text { Família é consultada para } \\
\text { saber se os serviços da USB } \\
\text { atendem aos problemas de } \\
\text { saúde dos usuários }\end{array}$ & $14,5(11,8-16,5)$ & - & - & - \\
\hline $\begin{array}{l}\text { Conhecimentos dos } \\
\text { profissionais sobre os } \\
\text { principais problemas de saúde } \\
\text { da comunidade }\end{array}$ & $25,7(22,2-28)$ & - & - & - \\
\hline $\begin{array}{l}\text { Oferta de serviços nas escolas } \\
\text { pela equipe da ESF }\end{array}$ & $12,1(9,9-14,3)$ & $49,4(37,4-60,1)$ & $75,0(56,5-88,5)$ & $<0,001^{*}$ \\
\hline $\begin{array}{l}\text { Frequência de visitas } \\
\text { domiciliares }\end{array}$ & $18,6(15,5-20,7)$ & $92,0(84,3-97,1)$ & $90,6(75-98)$ & $<0,001^{*}$ \\
\hline $\begin{array}{l}\text { Trabalho da USF com outras } \\
\text { instituições da comunidade } \\
\text { para realizar programas } \\
\text { intersetoriais }\end{array}$ & $15,5(12,6-17,4)$ & $40,0(29,2-51,5)$ & $62,4(43,6-78,8)$ & $<0,001^{*}$ \\
\hline $\begin{array}{l}\text { Realização de enquetes para } \\
\text { saber se os serviços oferecidos } \\
\text { estão respondendo às } \\
\text { necessidades da população }\end{array}$ & - & $11,2(5,2-20,2)$ & $43,7(26,3-62,3)$ & $<0,001^{*}$ \\
\hline $\begin{array}{l}\text { Realização de enquetes para } \\
\text { identificar problemas de saúde } \\
\text { da população }\end{array}$ & 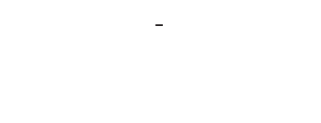 & $15,0(7-23,2)$ & $50,0(31,8-68,1)$ & $<0,001^{*}$ \\
\hline $\begin{array}{l}\text { Representação da comunidade } \\
\text { na direção da UBS ou nos } \\
\text { conselhos }\end{array}$ & $11,7(8,9-13,2)$ & $46,2(35-57,7)$ & $75,0(56,5-88,5)$ & $<0,001^{*}$ \\
\hline $\begin{array}{l}\text { Autonomia da USF para } \\
\text { organizar a oferta de serviços } \\
\text { de acordo com os problemas } \\
\text { da comunidade }\end{array}$ & - & $55,0(43,4-66,1)$ & $71,8(53,2-86,2)$ & 0,061 \\
\hline
\end{tabular}

*Teste Quiquadrado, há diferença entre os sujeitos se p $<0,05$. IC = Intervalo de Confiança 
Tabela 4. Escores dos indicadores e Índices Compostos do enfoque familiar e orientação para a comunidade na percepção dos usuários, profissionais e gestores da Estratégia Saúde da Família no município de São Luís - MA, 2011.

\begin{tabular}{|c|c|c|c|c|}
\hline Indicadores & $\begin{array}{l}\text { Escore } \\
\text { Usuários } \\
\text { (IC 95\%) }\end{array}$ & $\begin{array}{l}\text { Escore } \\
\text { Profissionais } \\
\text { (IC 95\%) }\end{array}$ & $\begin{array}{l}\text { Escore } \\
\text { Gestores } \\
\text { (IC 95\%) }\end{array}$ & $\begin{array}{l}\text { P-valor } \\
\text { Kruskal } \\
\text { Wallis }^{*}\end{array}$ \\
\hline \multicolumn{5}{|l|}{ Enfoque familiar } \\
\hline $\begin{array}{l}\text { Questionamento dos profissionais sobre fatores de } \\
\text { risco social ou condições de vida dos usuários }\end{array}$ & $2,4(2,2-2,7)$ & $4,6(4,3-4,9)$ & $5,2(4,8-5,5)$ & $<0,001$ \\
\hline $\begin{array}{l}\text { Questionamento sobre doenças de familiares durante } \\
\text { a consulta }\end{array}$ & $3,1(3,0-3,3)$ & $4,8(4,6-5,1)$ & $5,2(4,9-5,6)$ & $<0,001$ \\
\hline Conhecimento da família pelos profissionais & $2,3(2,5-2,7)$ & - & - & \\
\hline $\begin{array}{l}\text { Possibilidade do profissional discutir com a família } \\
\text { problemas de saúde dos usuários }\end{array}$ & $3,5(3,3-3,6)$ & $4,6(4,1-4,7)$ & $5,0(4,7-5,4)$ & $<0,001$ \\
\hline $\begin{array}{l}\text { Questionamento dos profissionais sobre ideias e } \\
\text { opiniões dos usuários acerca do seu tratamento }\end{array}$ & $2,1(1,9-2,2)$ & - & - & - \\
\hline $\begin{array}{l}\text { Organização dos prontuários médicos por família na } \\
\text { UBS }\end{array}$ & - & $5,6(5,4-5,8)$ & $5,9(5,8-6,0)$ & - \\
\hline Índice total do Enfoque Familiar & 2,7 & 4,9 & 5,3 & - \\
\hline \multicolumn{5}{|l|}{ Orientação para a comunidade } \\
\hline $\begin{array}{l}\text { Família é consultada para saber se os serviços da USB } \\
\text { atendem aos problemas de saúde dos usuários }\end{array}$ & $2,1(2,0-2,2)$ & - & - & - \\
\hline $\begin{array}{l}\text { Conhecimentos dos profissionais sobre os principais } \\
\text { problemas de saúde da comunidade }\end{array}$ & $3,3(3,1-3,4)$ & - & - & - \\
\hline Oferta de serviços nas escolas pela equipe da ESF & $2,8(2,7-3,0)$ & $4,4(4,1-4,7)$ & $5,2(4,8-5,6)$ & $<0,001$ \\
\hline Frequência de visitas domiciliares & $3,3(3,2-3,4)$ & $5,6(5,4-5,7)$ & $5,8(5,6-6,0)$ & $<0,001$ \\
\hline $\begin{array}{l}\text { Trabalho da USF com outras instituições da } \\
\text { comunidade para realizar programas intersetoriais }\end{array}$ & $3,0(2,9-3,2)$ & $3,9(3,5-4,2)$ & $4,5(4,0-5,0)$ & $<0,001$ \\
\hline $\begin{array}{l}\text { Realização de enquetes para saber se os serviços } \\
\text { oferecidos estão respondendo às necessidades da } \\
\text { população }\end{array}$ & - & $2,5(2,2-2,9)$ & $4,2(3,6-4,7)$ & - \\
\hline $\begin{array}{l}\text { Realização de enquetes para identificar problemas de } \\
\text { saúde da população }\end{array}$ & - & $2,7(2,4-3,0)$ & $4,2(3,7-4,8)$ & - \\
\hline $\begin{array}{l}\text { Representação da comunidade na direção da UBS ou } \\
\text { nos conselhos }\end{array}$ & $3,0(2,8-3,2)$ & $3,8(3,3-4,3)$ & $5,0(4,4-5,5)$ & $<0,001$ \\
\hline $\begin{array}{l}\text { Autonomia da USF para organizar a oferta de } \\
\text { serviços de acordo com os problemas da comunidade }\end{array}$ & - & $4,5(4,1-4,9)$ & $5,0(4,5-5,4)$ & - \\
\hline Índice total da Orientação para a Comunidade & 2,9 & 3,9 & 4,8 & - \\
\hline
\end{tabular}

nais e gestores essa coleta de informações e contato com familiares é, na maioria das vezes, uma realidade.

Na ESF a família deve ser entendida de forma integral e em seu espaço social, ou seja, a pessoa deve ser abordada em seu contexto socioeconômico e cultural, e reconhecida como sujeito social portadora de autonomia, sabendo que é na família que ocorrem interações e conflitos que influenciam diretamente na saúde das pessoas. Portanto, espera-se que os profissionais da saúde tenham compreensão de aspectos relacionados à dinâmica familiar, seu funcionamento, suas funções, desenvolvimento e características soci- ais, culturais, demográficas e epidemiológicas. Isso requer da equipe uma atitude diferenciada, pautada no respeito, na ética e no compromisso com as famílias pelas quais são responsáveis, mediante a criação de vínculo de confiança, atuando de forma participativa na construção de ambientes mais saudáveis no espaço familiar ${ }^{13}$.

$\mathrm{Na}$ análise dos profissionais e gestores uma alta porcentagem dos prontuários médicos está considerando o núcleo familiar. Entretanto, trabalhos mostram que inovações nos processos de trabalho e nas práticas dos profissionais de saúde, pretendidas pela ESF parecem ainda distantes de se efetivarem. Em estudo realizado na ci- 


\begin{tabular}{|c|c|c|c|c|}
\hline Indicadores & $\begin{array}{l}\text { P-valor } \\
\text { Mann } \\
\text { Whintey }^{* *}\end{array}$ & $\begin{array}{l}\text { P-valor } \\
\text { Pós-teste } \\
\text { Usuários } \\
\text { X Profissionais }\end{array}$ & $\begin{array}{l}\text { P-valor } \\
\text { Pós-teste } \\
\text { Usuários } \\
\text { X Gestores }\end{array}$ & $\begin{array}{l}\text { P-valor } \\
\text { Pós-teste } \\
\text { Profissionais } \\
\text { X Gestores }\end{array}$ \\
\hline \multicolumn{5}{|l|}{ Enfoque familiar } \\
\hline $\begin{array}{l}\text { Questionamento dos profissionais sobre fatores de } \\
\text { risco social ou condições de vida dos usuários }\end{array}$ & - & $<0,001$ & $<0,001$ & 0,250 \\
\hline $\begin{array}{l}\text { Questionamento sobre doenças de familiares durante } \\
\text { a consulta }\end{array}$ & - & $<0,001$ & $<0,001$ & 0,186 \\
\hline Conhecimento da família pelos profissionais & - & - & - & - \\
\hline $\begin{array}{l}\text { Possibilidade do profissional discutir com a família } \\
\text { problemas de saúde dos usuários }\end{array}$ & - & $<0,001$ & $<0,001$ & 0,110 \\
\hline $\begin{array}{l}\text { Questionamento dos profissionais sobre ideias e } \\
\text { opiniões dos usuários acerca do seu tratamento }\end{array}$ & - & - & - & - \\
\hline $\begin{array}{l}\text { Organização dos prontuários médicos por família na } \\
\text { UBS }\end{array}$ & 0,003 & - & - & - \\
\hline Índice total do Enfoque Familiar & - & - & - & - \\
\hline \multicolumn{5}{|l|}{ Orientação para a comunidade } \\
\hline $\begin{array}{l}\text { Família é consultada para saber se os serviços da USB } \\
\text { atendem aos problemas de saúde dos usuários }\end{array}$ & - & - & - & - \\
\hline $\begin{array}{l}\text { Conhecimentos dos profissionais sobre os principais } \\
\text { problemas de saúde da comunidade }\end{array}$ & - & - & - & - \\
\hline Oferta de serviços nas escolas pela equipe da ESF & - & $<0,001$ & $<0,001$ & 0,038 \\
\hline Frequência de visitas domiciliares & - & 0,007 & $<0,001$ & 0,127 \\
\hline $\begin{array}{l}\text { Trabalho da USF com outras instituições da } \\
\text { comunidade para realizar programas intersetoriais }\end{array}$ & - & 0,002 & $<0,001$ & 0,092 \\
\hline $\begin{array}{l}\text { Realização de enquetes para saber se os serviços } \\
\text { oferecidos estão respondendo às necessidades da } \\
\text { população }\end{array}$ & $<0,001$ & - & - & - \\
\hline $\begin{array}{l}\text { Realização de enquetes para identificar problemas de } \\
\text { saúde da população }\end{array}$ & $<0,001$ & - & - & - \\
\hline $\begin{array}{l}\text { Representação da comunidade na direção da UBS ou } \\
\text { nos conselhos }\end{array}$ & - & $<0,001$ & $<0,001$ & 0,003 \\
\hline $\begin{array}{l}\text { Autonomia da USF para organizar a oferta de } \\
\text { serviços de acordo com os problemas da comunidade }\end{array}$ & 0,022 & - & - & - \\
\hline Índice total da Orientação para a Comunidade & - & - & - & - \\
\hline
\end{tabular}

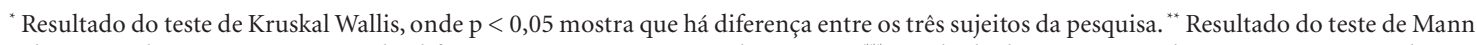
Whitney, onde $\mathrm{p}<0,05$ mostra que há diferença entre os três sujeitos da pesquisa. ${ }^{* * *}$ Resultado do pós-teste, onde $\mathrm{p}<0,05$ mostra que há diferença entre os grupos. IC= Intervalo de Confiança.

dade do Rio de Janeiro por Vasconcelos et al. ${ }^{14}$, que avaliava a qualidade dos prontuários na atenção básica, os autores encontraram uma pequena presença do registro sobre as condições de situação familiar que, além de ser variável fundamental também para acompanhamento clínico individual, é estratégica em áreas de atuação de programas que têm a família como objeto. Portanto, o prontuário da família é elemento decisivo à melhor atenção à saúde dos seus membros e precisa reunir as informações necessárias à continuidade do acompanhamento prestado à família, considerando o território onde habita e as condições de vida dos seus membros.
A realização de visitas domiciliares como uma prática constante nas atividades realizadas pela equipe de saúde também não teve uma avaliação satisfatória pelos usuários, como foi observado para os outros dois grupos estudados. Os dados encontrados corroboram com Albuquerque e Bosi $^{15}$, que evidenciaram que, apesar de ser uma atividade programada e inserida no cronograma, a visita domiciliar é ainda percebida, pelos usuários, como algo esporádico, quando realizada. Outra pesquisa, que analisou a concepção da equipe de saúde da família sobre as visitas domiciliares ${ }^{16}$, mostrou que os profissionais consideram as visitas atividades potenciais para se 
utilizar tecnologias leves e proporcionar novos modos de se cuidar na saúde; mais humanas e acolhedoras, envolvendo efetividade e laços de confiança entre os profissionais, os usuários, a família e a comunidade.

Outro indicador que apresentou uma avaliação insuficiente na visão dos usuários foi a intersetorialidade, sendo inclusive essa uma informação desconhecida para um significativo número de usuários entrevistados. Paula et al. ${ }^{17}$ destacam a importância da intersetorialidade por ser um dos elementos centrais para a operacionalização da APS nos serviços de saúde, compreendendo-a como a capacidade de articular os vários setores presentes, também no nível mais operacional, local. Neste sentido, as ações de saúde são ofertadas à população com uma dimensão mais voltada ao planejamento e com potencialidade de articular setores fundamentais para desencadear mudanças mais efetivas e duradouras para o setor saúde.

Dos indicadores avaliados com os usuários no atributo da orientação para a comunidade três destes (estímulo da SEMUS para a participação social em reuniões de direção da USF ou conselho local; a oferta de serviços nas escolas pela equipe da ESF e a intersetorialidade, discutida no parágrafo anterior) tiveram uma alta porcentagem de "não sabe" como resposta. Isso levanta a discussão sobre a falta de conhecimento da comunidade acerca das atividades realizadas na ESF e a incipiente participação da mesma nos processos democráticos. Podendo, também, ser questionada a quase inexistência dessas ações na realidade da ESF no município de São Luís.

A participação social é um processo de aprendizagem que se exerce no respeito às diferenças e na ampliação dos espaços de convivência e debate político. Ao mesmo tempo em que é alimentada pela matriz cultural de uma sociedade é, também, a possibilidade de transformá-la. A valorização de espaços participativos pode ser um caminho para o aprimoramento democrático via incorporação de segmentos, até então fora da possibilidade de formularem e interferirem na execução de políticas pública ${ }^{18}$.

Este estudo tem como pontos fortes ser de base populacional e ter sido desenhado para com- parar respostas de gestores, profissionais e usuários. Tem como limitação a mais baixa taxa de participação de gestores e profissionais do que de usuários.

\section{Conclusões}

A avaliação geral do enfoque familiar e da orientação para a comunidade como atributos da APS na ESF foram insatisfatórias, principalmente na percepção dos usuários. Já os gestores atribuíram melhores percentuais em quase todos os indicadores estudados, o que pode ser entendido como um viés de intenção uma vez que eles podem avaliar positivamente as unidades que coordenam. As diferenças de percepções entre os três grupos de sujeitos pesquisados foram estatisticamente significantes, e essas divergências estão principalmente entre usuários e profissionais e usuários e gestores.

Uma significativa porcentagem dos usuários informou não saber responder várias questões sobre os indicadores do atributo orientação para a comunidade, como estímulo da SEMUS para a gestão participativa, oferta de serviços nas escolas e intersetorialidade, o que nos leva a pensar na falta de envolvimento da população nas atividades realizadas pela equipe da ESF e/ou a quase inexistência dessas ações.

A realização de enquetes com os usuários para saber se suas necessidades e as da comunidade estão sendo atendidas está entre os indicadores com pior avaliação, o que nos mostra que os princípios básicos da ESF, que devem ter como foco prioritário a família dentro das singularidades do ambiente em que está inserida, não estão sendo totalmente seguidos. Isto pode refletir ainda a continuidade de uma atenção pautada no modelo individual e curativo.

Tendo em vista que a ESF é o programa de qualificação da APS de maior sucesso da história do Brasil, espera-se que as informações desta pesquisa possam contribuir para mudanças na atenção oferecida pelas equipes em São Luís, trazendo discussões sobre a realização de um trabalho que tenha como prioridade o núcleo familiar e a comunidade dentro de suas reais necessidade. 


\section{Colaboradores}

MN Alencar, LC Coimbra e SRA Pinheiro participaram na concepção geral, pesquisa e redação final; AAM Silva e RCS Queiroz na redação final e APP Moraes na revisão.

\section{Agradecimentos}

Agradeço à Fundação de Amparo à Pesquisa e ao Desenvolvimento Científico e tecnológico do Maranhão pelo financiamento desta pesquisa. E ao Programa de Pós-Graduação em Saúde Coletiva da Universidade Federal do Maranhão.

\section{Referências}

1. Souza MF, Hamann EM. Programa Saúde da Família no Brasil: Uma agenda incompleta. Cien Saude Colet 2009; 14(Supl. 1):1325-1335.

2. Campos CEA. Estratégias de avaliação e melhoria contínua da qualidade no contexto da Atenção Primária à Saúde. Rev Bras Saude Materno Infantil 2010; 5(Supl. 1):S63-S69.

3. Starfield B. Atenção primaria: equilíbrio entre necessidades de saúde, serviços e tecnologia. Brasília: UNESCO, Ministério da Saúde (MS); 2004.

4. Felisberto E. Monitoramento e avaliação da atenção básica: novos horizontes. Rev Bras Saude Materno-Infantil 2004; 4(3):317-321.

5. Brousselle A, Champagne R, Contrandriopoulos A, Hartz Z. Avaliação: conceitos e métodos. Rio de Janeiro: Editora Fiocruz; 2011.

6. Ibañez N, Rocha LSY, Castro PC, Ribeiro MCS, Foster AC, Novaes MHD, Viana ALD. Avaliação do desempenho da atenção básica no estado de São Paulo. Cien Saude Colet 2006; 11(Supl. 3):683-703.

7. Elias PE, Ferreira CW, Alves MCG, Cohn A, Kishima Júnior AE, Gomes A, Bousquat A. Atenção básica em saúde: comparação entre PSF e UBS por estrato de exclusão social no município de São Paulo. Cien Saude Colet 2006: 11(3):633-641.

8. Van Stralen CJ, Belisário SA, Van Stralen TB, Lima AMD, Massote AW, Oliveira CL. Percepção dos usuários e profissionais de saúde sobre atenção básica: comparação entre unidades com e sem saúde da família na região Centro-Oeste do Brasil. Cad Saude Publica 2008; 24(Supl. 1):148-158.

9. Brasil. Ministério da Saúde (MS). Cadastro Nacional de Estabelecimentos de Saúde [homepage na internet]. Número de equipes de Saúde da Família. [acessado 2011 maio 22]. Disponível em: http:// cnes.datasus.gov.br/

10. Almeida CA, Macinko J. Mecanismos institucionais de monitoramento e avaliação da atenção básica. In: Organização Pan-americana de Sáude (OPAS), Ministério da Saúde (MS), organizadores. Validação de uma metodologia de avaliação rápida das características organizacionais e do desempenho dos serviços de atenção básica do Sistema Único de Saúde (SUS) em nível local. Brasília: OPAS, MS; 2006. (Série Técnica Desenvolvimento de Sistemas e Serviços de Saúde)

11. Brasil. Ministério da Saúde (MS). Manual do instrumento de avaliação da atenção primária à saúde. Brasília: MS; 2010.

12. Nogueira JA. Enfoque familiar e orientação para a comunidade no controle da tuberculose. Rev Bras Epidemiol 2011; 14(Supl. 2):207-216.

13. Brasil. Mistério da Saúde (MS). Secretaria de Políticas Públicas. Guia prático do Programa de Saúde da Família. Brasília: MS; 2001. 
14. Vasconcelos MM, Gribel EB, Moraes IHS. Registros em saúde: avaliação da qualidade do prontuário do paciente na atenção básica, Rio de Janeiro, Brasil. Cad Saude Publica 2008; 24(Supl. 1):173-182.

15. Albuquerque ABB, Bosi MLM. Visitas domiciliares no âmbito da Estratégia Saúde da Família: percepções de usuários no município de Fortaleza, Ceará, Brasil. Cad Saude Publica 2009; 25(Supl. 5):1103-1112.

16. Sakata KN, Almeida MCP, Alvarenga AM, Craco PF, Pereira MJB. Concepções da equipe de saúde da família sobre as visitas domiciliares. Rev Bras Enferm 2007; 60(Supl. 6):659-664.

17. Paula KA, Palha PF, Protti ST. Intersetorialidade: uma vivência prática ou um desafio a ser conquistado? Interface (Botucatu) 2004; 15(Supl. 8):331-348.

18. Escorel S, Moreira MR. Participação social. In: Giovanella L, Escorel S, Lobato LVC, Noronha JC, Carvalho AI, organizadores. Políticas e sistemas de saúde no Brasil. Rio de Janeiro: Ed. Fiocruz; 2008.

Artigo apresentado em 14/04/2013

Aprovado em 26/05/2013

Versão final apresentada em 04/06/2013 